The Association

ACAMI for Child and Adolescent

Mental Health

तूล

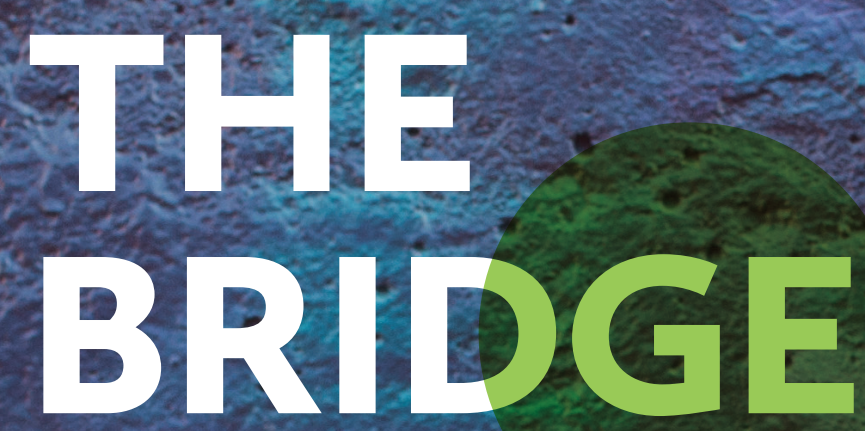

April 2018

Gender

Identity

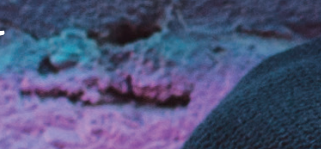

Practitioner Review:

Affirmative care may.

elicit the best mental-

health outcomes in

transgender youths

Research highlights

from our journals -

JCPP and CAMH

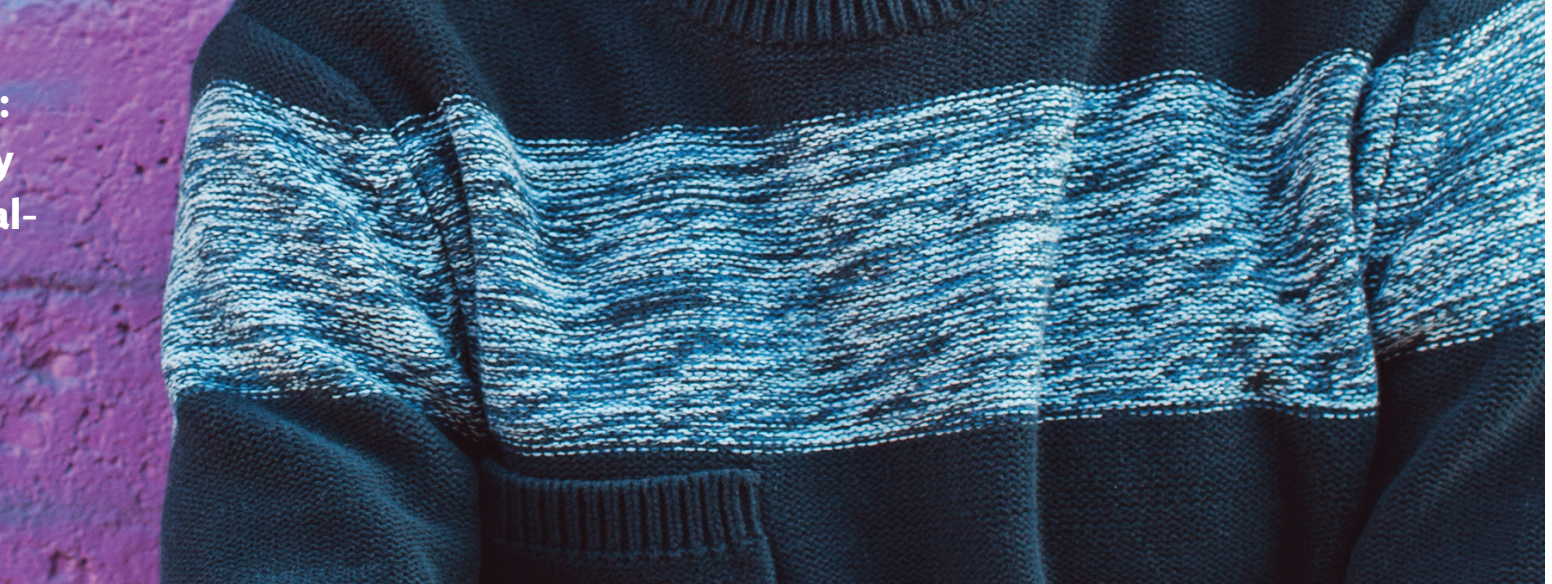

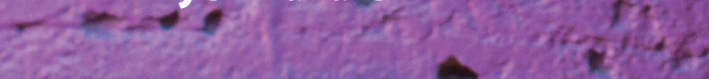

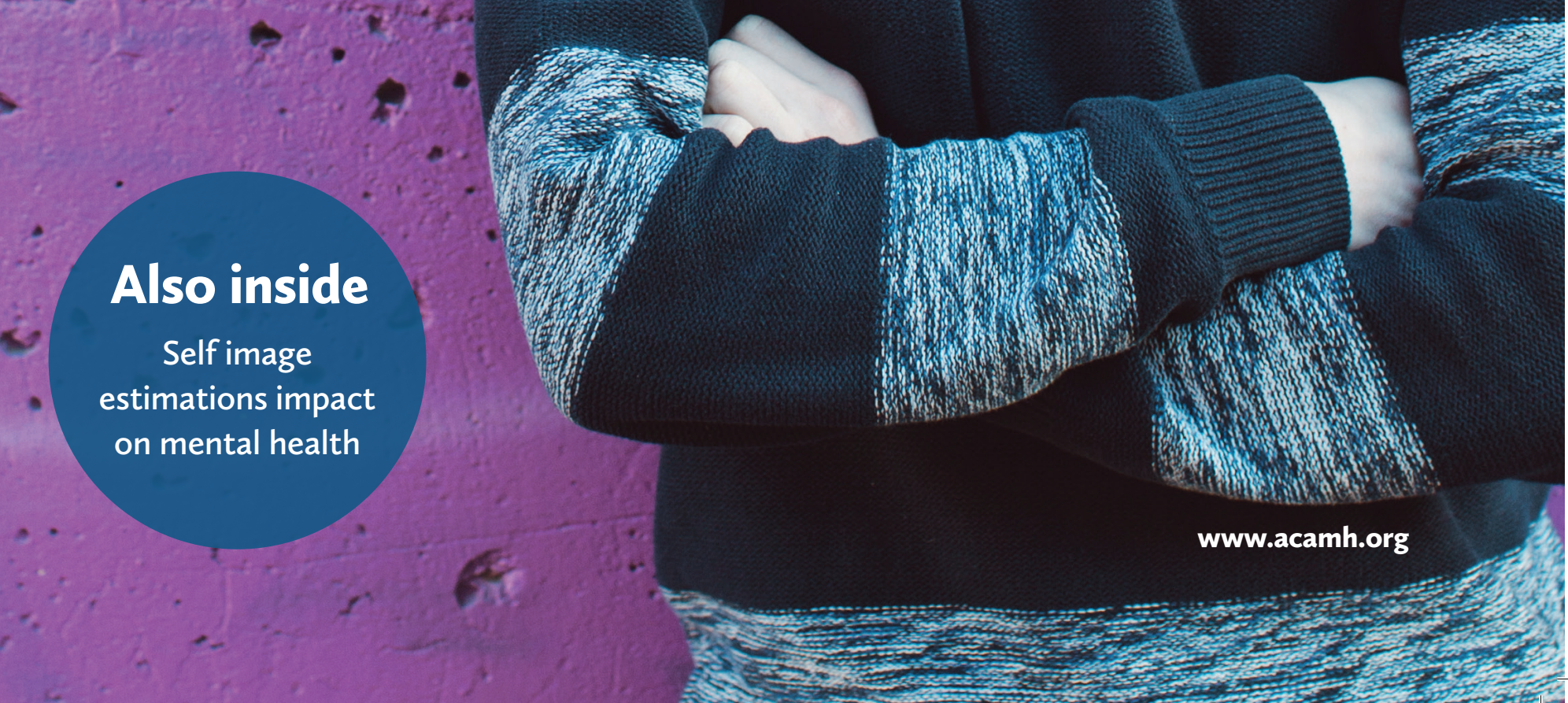




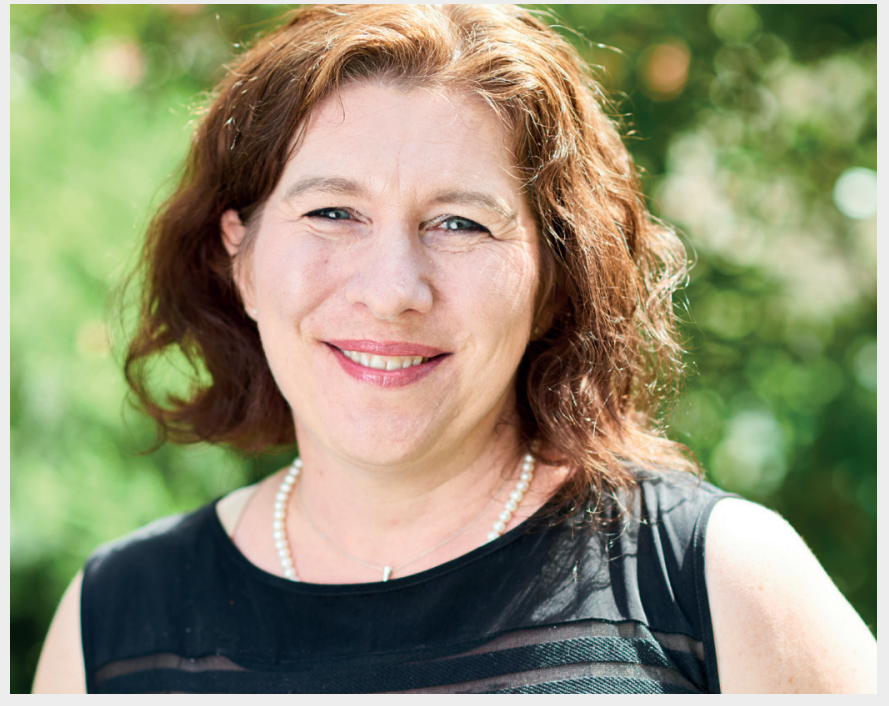

\section{Foreword from the Editor}

\section{Dr Juliette Kennedy}

Editor of The Bridge

Welcome to The Bridge - this month focusses on gender diversity in children and young people. Turban et al research review in JCCP 2017 is summarised here. Gender concern in young people can be associated with a great deal of distress; Turban et al propose that external social reactions, rather than internal conflicts, may be the primary cause of distress/depression/anxiety, that may then be linked to self-harm risk. It is highlighted that support and acceptance of gender variance, by family and friends and protection from bullying and discrimination, are crucial in preventing mental distress and the potential emergence of mental health problems in young people with gender concerns. The support and campaigning work of Mermaids and Gendered Intelligence is also described here. Young people and families whom I have worked with, have found that contact with other young people in a similar position, or further ahead in their journey, very helpful and supportive. Both organisations also provide information and advice to professionals working with young people and have a lot of information on their websites. I hope you find this edition helpful.

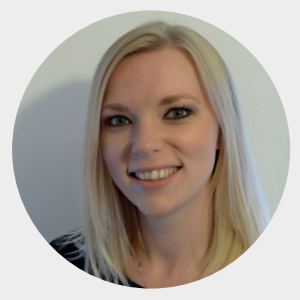

All of the research highlights in this edition are prepared by $\mathrm{Dr}$ Jessica $\mathrm{K}$ Edwards. Jessica is a freelance editor and science writer, and started writing for 'The Bridge' in December 2017.

\section{Elevated} anxiety risk in sexual minority adolescents

Adolescents that are not exclusively heterosexual ("sexual minority") are at an increased risk of developing an anxiety disorder compared to their heterosexual counterparts, according to recent data from a prospective birth cohort study. The study, conducted by researchers at King's College London, investigated the relationship between sexual orientation at 15.5 years-of-age and the development of an anxiety disorder by 17.5 years-of-age in a large cohort of $>4,500$ young people. The results showed that more sexual minority adolescents experienced early childhood gender nonconformity (CGN), low self-esteem and incidents of bullying from ages 12-16 years compared to exclusively heterosexual adolescents. Consequently, those identifying as sexual minority at 15.5 years-of-age had $\sim 2.5$ times higher risk of developing an anxiety disorder than those identifying as heterosexual; bullying and low self-esteem were found to be key contributing factors. Interestingly, CGN had little effect on the association between sexual orientation and risk of developing an anxiety disorder. The researchers propose that interventions for anxiety disorders in sexual minority adolescents should consider prior experiences of bullying and low self-esteem.

Jones, A., Robinson, E., Oginni, O., Rahman, $Q$. \& Rimes, K. A. (2017), Anxiety disorders, gender nonconformity, bullying and self-esteem in sexual minority adolescents: prospective birth cohort study. J. Child Psychol. Psychiatr. 58, 1201-1209. doi:10.1111/ jcpp.12757 


\section{Mermaids - helping gender diverse young people}

\section{By Louie Sandys, Editorial Assistant, ACAMH}

Mermaids, is a charity that supports children and young people up to 20 years old who are gender diverse, alongside their families and professionals involved in their care. They do this by raising awareness of gender nonconformity in children, young people and amongst professionals and the public. A 2012 survey by the Equality and Human Rights Commission indicated that $1 \%$ of the 10,000 people who undertook the survey were experiencing gender variance.

Therefore, Mermaids campaign for the recognition of gender dysphoria in young people. Lobbying for improvements in professional services is sorely needed. Their hard work and the impact on the lives of many has been recognised through a number of accolades including the Community Group of the Year Award at the 2016 National Diversity Awards.

Mermaids was formed in 1995 by parents who were united by their children's longstanding gender incongruence. It has been a work in constant progress as Mermaids has grown to meet huge surges in demand offering the most appropriate resources to young people and their families, as well as carers and professionals that work with gender variant young people. Over the last few years, Mermaids has been able to widen their focus from its primary emphasis on being a support group.

Their website holds a wealth of resources for both children and parents, support with managing prejudice and hate crime, to useful videos, podcasts and factsheets. It's also a great resource for young people or parents that want to find out more.
Mermaids also run residentials to help connect and offer a space for young people and their parents to meet in person and also to meet role models who have been through their own gender variance. This has offered a great source of help, friendship and support to people worried about what they are experiencing and what the future holds.

One especially powerful resource has been achieved through the use of the forums. There is a forum for young people aged 12-19 years old, providing a safe space to talk to peers and make new friends with similar experiences. Likewise, there is a forum for parents; the impact of which can be seen in the Parents Voices section, highlighting what a difference it makes finding other parents who are going through the same journey.

The Young Voices section, also holds a number of very emotive and powerful poems and experiences written by children from as young as 7 , through to young adults who joined the community at Mermaids in their own childhood/ teenage years. These testimonials highlight the importance of the organisation's work. It is important to ensure more funding is made available to help others and ensure the wider public are aware of gender incongruence.

For more information on Mermaids and to get in contact with the team, please visit their website at: www.mermaidsuk.org.uk

\section{Key points:}

- Mermaids seeks to reduce the isolation and loneliness that parents and young people experiencing gender issues often face.

- They do this by empowering families and young people with the tools they need to negotiate education and health services.

- It is hoped that this support will help to reduce isolation and suicidality and self-harm in the young people who get in touch with Mermaids and also equip their parents to support their children.

- Mermaids aspires to improve self-esteem and social functioning in young people suffering with gender issues.

- Mermaids helps to improve awareness, understanding of GP's, CAMHS, Social Services and other professionals. 


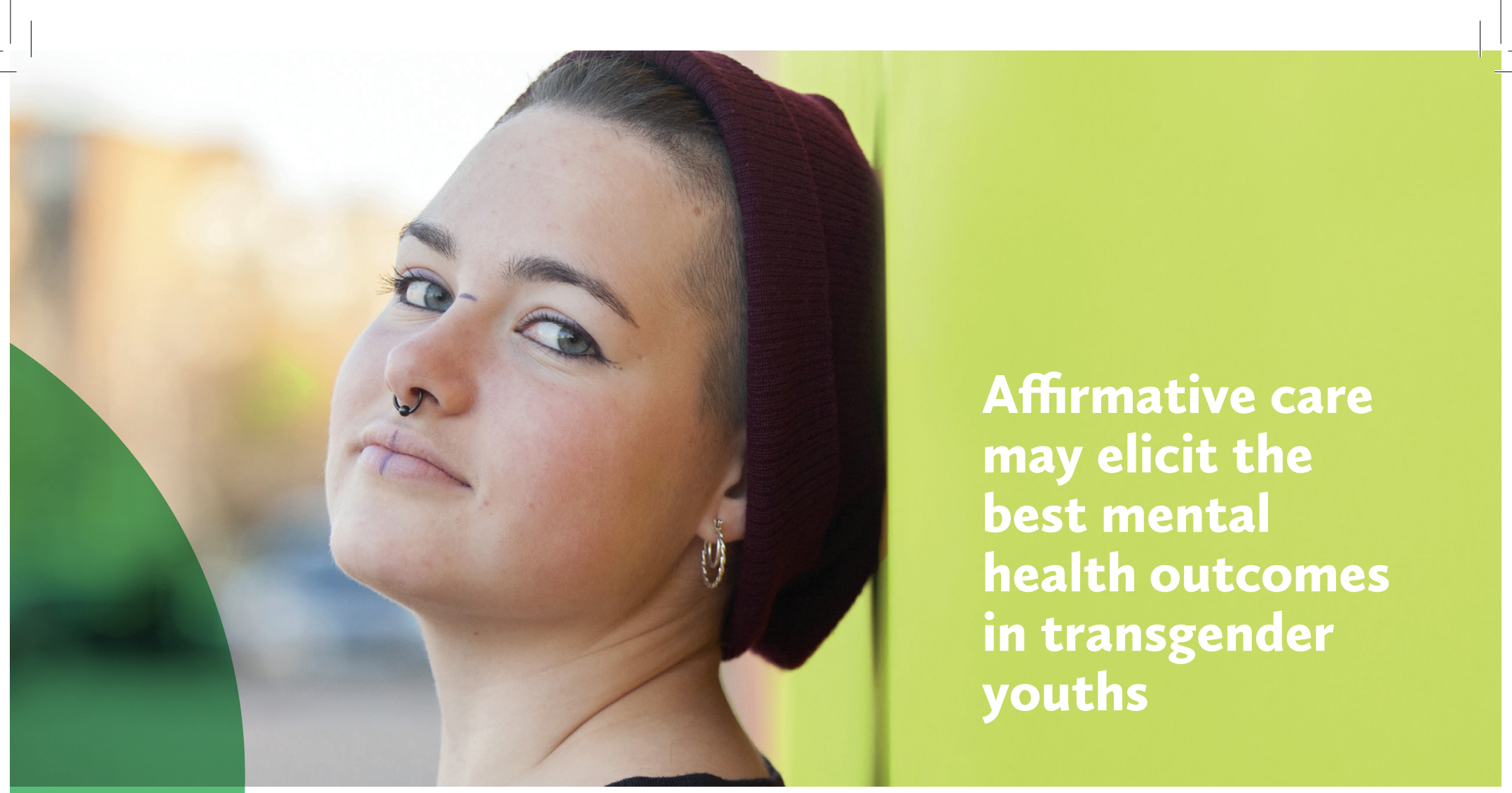

\section{The past decade has seen a marked surge in public interest in the issues surrounding gender dysphoria, as indicated by an increasing number of published articles in the medical literature, and media and political attention.}

By Dr. Jessica K. Edwards

In their recent review published in The Journal of Child Psychology and Psychiatry, Jack Turban and Diane Ehrensaft provide an update on how the field of pediatric gender identity is changing and discuss the approaches to supporting and managing the gender identity concerns of children and adolescents. They highlight that high rates of anxiety, depression and suicidal intentions in children with gender concerns may be reduced by following affirmative treatment protocols. Issues surrounding gender identity have been in the public limelight over recent years, and this attention has been matched with an exponential increase in research articles published on the topic. In 2005, <10 new articles focusing on transgender youths were indexed in PubMed; conversely, >200 new articles were published in 2017 alone. Consistently, the number of referrals to pediatric clinics specializing in gender identity has also increased. "We set out to review this bank of literature and provide a succinct update for mental-health professionals treating children and adolescents", says Turban. "Our main questions were: (1) what has the field learned about the epidemiology and mental-health concerns for transgender youths, (2) is there a model of care that is most robust for treating gender diverse children and adolescents, and (3) do we have evidence to support the use of medical interventions (such as puberty blockers and cross-sex hormones) in adolescents who are asserting a transgender identity?"

The researchers first analyzed the prevalence of transgender identification. One study by The Williams Institute (UCLA) published in 2016 estimated that there are $\sim 1.4$ million transgender adults residing in the USA, which accounts for $\sim 0.6 \%$ of the population. Although the studies in children have been relatively less comprehensive than those involving adults, the reported prevalence of transgender identification among adolescents is similar to adults. For example, studies of school children residing in San Francisco and New Zealand found that $1.3 \%$ and $1.2 \%$ of adolescents, respectively, identified themselves as transgender. The researchers conclude that this overall prevalence rate ( $1 \%$ young people) is much higher than previously thought.

Turban and Ehrensaft did, however, identify some limitations incurred by many studies estimating the prevalence of transgender identity in youths. First, the research field is limited by shifting terminology regarding cross-gender identification, which makes interpretation and comparisons between studies difficult. Second, many older studies relied on the child behavior checklist $(\mathrm{CBCL})$, which asks the participants only two questions 


\section{"Family support, therefore, seems to be a critical buffer in reducing the psychiatric risk factors that many transgender and gender diverse children and adolescents experience".}

related to gender identity and records the answers using a scale of o (never), 1 (sometimes) and 2 (often). Such method of reporting can be unreliable, as some children transiently "wish" to be of the opposite sex (perhaps because they have interests associated with the opposite gender), but do not actually have a true core transgender identity. More recent studies, however, have used more relevant and direct questions such as "do you identify as transgender?" Third, there are strong societal pressures to conceal gender dysphoria in order to avoid the associated stigma. As such, prevalence values of transgender youths are estimates at best.

Turban et al also investigated the cooccurring conditions with transgender identity and found that transgender youths suffer high rates of anxiety and depression. In fact, estimates for mood disorders range from $12.4 \%$ to $64 \%$, and estimates for anxiety disorders range from $16.3 \%$ to $55 \%$. Much of these data have come from chart reviews of youths referred to gender clinics. Consistently, a study of 105 gender dysphoric adolescents in the Netherlands found similar prevalence rates of internalizing emotional difficulties: $12.4 \%$ suffered from mood disorders and $21 \%$ suffered from anxiety disorders. The wide range in prevalence may be due to differences in parameters such as age, diagnostic criteria and cultural beliefs. What does seem to be clear is that internalizing emotional difficulties worsen with age.
By contrast, two studies involving transgender youths who were supported by their families (and who were not included in clinic samples) found no significant differences in the incidence of internalizing emotional difficulties compared to non-transgender control participants. "Non-acceptance by family and peers is a major risk factor for such mental-health problems in this population", describes Turban. "Family support, therefore, seems to be a critical buffer in reducing the psychiatric risk factors that many transgender and gender diverse children and adolescents experience". Some have also suggested that bullying and victimization contributes to mental-health problems in transgender youths, with an estimated $80 \%$ of transgender children being the victims of bullying. As such, Turban and Ehrensaft propose that social reactions, rather than internal conflicts, could be the primary cause of internalizing emotional difficulties in these children.

Data identified by this review also show high suicide attempt rates in the transgender population. Affected adults have a lifetime suicide rate of $\sim 41 \%$, and chart review studies from various pediatric gender identity clinics have found suicide attempt rates ranging from $9.3 \%$ (by mean age 14.8 years) and $10 \%$ (by mean age 13.5 years) up to $30 \%$ (by mean age 19.2 years). Children as young as 5 years old are at risk of suicidality, and the risk increases with age. Again, Turban et al found that non- acceptance by family members of youths with transgender identity has a strong correlation with suicidality. Finally, some studies have suggested a link between autism spectrum disorders (ASD) and gender dysphoria. Turban et al highlight, however, that many of the studies suggesting this etiological link between autism and gender dysphoria have limitations and as yet, no correlation between the two conditions has been proven.

The researchers also assessed the psychotherapeutic approaches and interventions available to pre-pubertal youths. They found emerging data to support that early affirmation with a non-pharmacological social transition (i.e. allowing a child to take on a name, pronouns, dress, etc. associated with their gender identity) results in good outcomes for pre-pubertal transgender children who wish to transition.

In addition, early data also suggest that puberty blockers and cross-sex hormones result in improved mental health outcomes for transgender adolescents.

Turban, J. L. \& Ehrensaft, D. Research Review: Gender identity in youth: treatment paradigms and controversies. (2017) J Child Psychol and Psychiatr. doi:10.1111/jcpp.12833. 


\section{Gendered Intelligence - helping young trans people}

By Dr Jay Stewart 


\section{Self image estimations impact on mental health}

Our overall estimation of "self image" is thought to be based on various parameters, such as perceptions of body image, mental function and social attitude. Now, a study by Maria Di Blasi and colleagues has investigated the extent by which these different parameters can influence psychological well-being. The researchers asked 128 adolescents (aged 13-18) seeking psychotherapeutic treatment to complete two questionnaires: the revised Offer Self-Image Questionnaire (revised) and the Youth-Outcome Questionnaire, which assess self image and psychological distress, respectively. Here, they found that the level by which adolescents cope with internal and external demands (their "impulse control"), maintain mood and emotional fluctuations (their "emotional tone"), and perceive the quality of their family and/or peer relationships were negative predictors of psychological distress. Gender differences in terms of risk of psychological distress were identified in this cohort, suggesting that gender may influence mental-health outcomes. Specifically, a stronger negative association was found between impulse control and psychological distress in boys, and between both body-image satisfaction and family relationships and psychological distress in girls. The researchers propose that examining maladaptive psychological components of self image may help predict risk of psychological impairment and guide the development of effective mentalhealth interventions.

Di Blasi, M., Muccioli, P., Alagna, M., Torres, D., Duca, I. \& Tosto, C. (2017), Self-image and psychological distress in treatment-seeking adolescents. Child Adolesc Ment Health. doi:10.1111/ camh.12255 


\section{Jack Tizard Memorial}

\section{Lecture \& Conference}

'Expert analysis, new research: what works' Intellectual Disability: 7 June, Autism: 8 June

The Royal College of Physicians, London

Under the theme 'Expert analysis, new research: what works' the first day will concentrate on Intellectual Disability, whilst the second will be on Autism. You will hear from world-leading experts in the field of Intellectual Disability, and Autism, delivering the latest evidence and best practice examples, that will shape people's knowledge. Visit www.acamh.org for full details.

The National Autistic Society

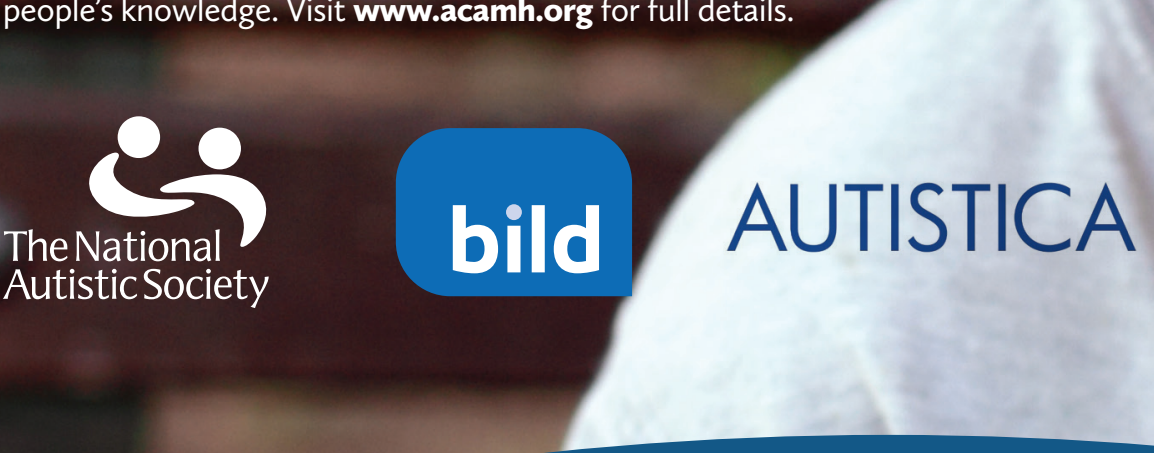

\section{Processing speed determines dyslexia risk}

Males exhibit a lower average reading performance than females, according to new data from Anne Arnett and colleagues. The researchers devised a framework to first validate the apparent sex difference in prevalence of dyslexia and then determine which cognitive correlates may underlie this difference. The study included 2,401 youths aged 7-24 years, who were asked to complete tests for reading accuracy and IQ. Analysis of the results verified that males have a lower mean and more variable reading performance than females. This difference may be due to a slower processing speed (PS; the time required to perform a mental task) and poorer inhibitory control (INH; the capacity to regulate a behavioural response) demonstrated by males compared to females in this cohort. Males did, however, perform better than females in verbal reasoning (VR; the ability to comprehend concepts expressed in words). The researchers conclude that reading performance in males is associated with two cognitive risk factors (PS and INH) and a protective factor (VR), which together mediate the sex bias towards males in dyslexia.

Arnett, A. B., Pennington, B. F., Peterson, R. L., Willcutt, E. G., DeFries, J. C. \& Olson, R. K. (2017), Explaining the sex difference in dyslexia. J Child Psychol Psychiatr, 58:719-727. doi:10.1111/jcpp.12691

\section{Rumination affects mother-infant interactions}

Rumination - or focused attention on the symptoms of one's distress - is associated with the onset and maintenance of depression, but whether it affects the quality of interaction between an infant and a mother affected by postnatal depression is unclear. New research now shows that a ruminative state can affect the quality of sensitive maternal behaviours. A total of 79 mothers, of whom half exhibited depressive-like symptoms (dysphoria), were randomly assigned to a "rumination induction" or control group. The rumination induction group were asked to indentify unresolved, selfrelevant problems and the control group were asked to identify resolved, self-relevant problems. Each group focused on these problems for 10 minutes, and mother-infant interactions were analyzed. Dysphoric mothers showed reduced quality of interaction with their infants compared to the non dysphoric controls, and mothers in the rumination induction group exhibited reduced sensitivity towards their infants compared to those in the control group. The researchers conclude that a ruminative state impairs the quality of mother-infant interaction, regardless of the initial level of depressive symptoms the mother is experiencing.

Tester-Jones, M., Karl, A., Watkins, E. \& O'Mahen, H. (2017), Rumination in dysphoric mothers negatively affects motherinfant interactions. J Child Pyschol Psychiatr. 58: 38-45. doi:10.1111/jcpp.12633 